\title{
Culture media for detection of Acinetobacter baumannii selective media for detection of $A$ baumannii
}

\begin{abstract}
Detection of Acinetobacter baumannii is of crucial importance to the prevention of the development of nosocomial infections. CHROMagar Acinetobacter ${ }^{\circledR}$ is a new selective medium recently developed for the rapid identification of $A$. baumannii. This medium incorporates enzymatic substrates that enable colour-based preliminary identification of bacterial colonies recovered within 18 to $24 \mathrm{~h}$ of inoculation. Sheep blood agar (SBA) supplemented with ertapenem contains few modifications and has not yet been defined in the literature. The aim of this study was to evaluate CHROMagar Acinetobacter ${ }^{\circledR B}$ for detection of $A$. baumannii and compare its performance to that of SBA supplemented with ertapenem.

A total of 73 Gram-negative bacteria were evaluated. A. baumannii, Pseudomonas aeruginosa, Stenotrophomonas maltophilia and carbapenemase-positive Klebsiella pneumoniae were grown on CHROMagar Acinetobacter ${ }^{\circledR}$ and supplemented SBA media. Susceptible clinical isolates and standard strains of Enterobacteriaceae were inhibited by both CHROMagar Acinetobacter ${ }^{\mathbb{B}}$ and supplemented SBA. C. albicans isolates, inoculated with mixed samples, did not grow on CHROMagar Acinetobacter ${ }^{\circledR}$ after 24 or $48 \mathrm{~h}$, but did grow on supplemented SBA after $24 \mathrm{~h}$ and exhibited distinct colony morphology. $A$. baumannii, the most clinically relevant of the bacteria tested, have a particular propensity for nosocomial transmission, due in part to their sustained survival on environmental surfaces as well as their multidrug resistance. The prevalence of infection with $A$. baumannii has increased significantly during the last decade. Due to well-designed solid selective culture media are required for detection of Acinetobacter spp. We advise the use of supplemented SBA and CHROMagar Acinetobacter ${ }^{\circledR}$ medium for rapid detection of nosocomial infection in the absence of a confirmatory procedure. Investigation into the metabolism of $A$. baumannii will facilitate the development of chromogenic media.
\end{abstract}

Volume 2 Issue 3 - 2015

\author{
Aysenur Yagmur Ciftci,' Engin Karakece, ${ }^{2}$ \\ Ali Riza Atasoy, ${ }^{2}$ Gulsah Asik, ${ }^{3}$ Ihsan Hakki \\ Ciftci $^{2}$ \\ 'Sakarya Cevat Ayhan Science High School, Turkey \\ ${ }^{2}$ Department of Medical Microbiology, Sakarya University, Turkey \\ ${ }^{3}$ Department of Medical Microbiology, Afyon Kocatepe \\ University, Turkey
}

\begin{abstract}
Correspondence: Engin Karakece, Department of Medical Microbiology, Sakarya University, School of Medicine, Sakarya, Turkey, Tel +9026425521 I0, Fax +90264255202,
\end{abstract} Email enginkarakece@gmail.com

Received: March 12, 2015 | Published: May 14, 2015

Keywords: Acinetobacter baumanni, CHROMagar, selective medium

Abbreviations: MDRAB, multi drug-resistant A. baumannii; SBA, sheep blood agar; PCR, Pseudomonas aeruginosa carbapenem-susceptible and resistant; KPC, Klebsiella Pneumoniae carbapenemase; CFU, colony forming units, CHR, CHROM agar Acinetobacter, $\mathrm{CHR}^{+}, \mathrm{CHROM}$ agar acinetobacter supplemented with 400mg/L KPC; $\mathrm{CHR}^{++}$, CHROM agar acinetobacter supplemented with $800 \mathrm{mg} / \mathrm{L} \mathrm{KPC}$; $\mathrm{SBA}^{+}$, sheep blood agar supplemented with $2 \mathrm{mg} / \mathrm{L}$ ertapenem

\section{Introduction}

Members of the genus Acinetobacter have become the focus of much research due to their pathogenic potential. Several species persist in hospital environments and cause severe life-threatening infections in immunocompromised patients. ${ }^{1}$ Particularly, Acinetobacter baumannii is recognised as an increasingly important opportunistic pathogen among such species. ${ }^{2} A$. baumannii have the ability to develop resistance to new antibiotics extremely rapidly. Many clinical isolates of $A$. baumannii are now resistant to all conventional antimicrobial agents including carbapenems. ${ }^{3}$ Today, in many parts of the world strains resistant to almost all available antimicrobial classes (multidrug-resistant A. baumannii or MDRAB) have been reported. These organisms have been implicated in a diverse range of infections and are particularly problematic in intensive care units. ${ }^{4}$ Moreover, detection of $A$. baumannii carriers and the establishment of isolation measures are of crucial importance to preventing the development of nosocomial infections due to A. baumannii.

Several selective and differential media are available for the isolation of Acinetobacter spp. One such medium incorporates bile salts and bromocresol purple. Another contains bile salts, ampicillin, cefsulodin, and vancomycin. In the Leeds medium formulation, ampicillin was removed and the concentrations of the other antibiotics were adjusted to optimise performance. ${ }^{5}$ CHROMagar Acinetobacter ${ }^{\mathbb{B}}$ (CHROMagar, Paris, France) was recently developed as a selective agar for the rapid identification of $A$. baumannii. It incorporates enzymatic substrates, enabling colour-based preliminary identification of colonies recovered within 18 to $24 \mathrm{~h}$ of inoculation. This medium also contains agents that inhibit the growth of most Gram-positive and negative organisms. Furthermore, CHROMagar Acinetobacter ${ }^{\mathbb{R}}$ agar supplemented with KPC suppresses carbapenem-susceptible Gram-negative bacilli ${ }^{3}$. Sheep blood agar (SBA) can be modified and supplemented with common antibiotics for strains that are resistant to for example, ertapenem. Additionally, supplemented SBA has not been used for detection of $A$. baumannii in the literature.

The aim of this study was to evaluate CHROMagar Acinetobacter ${ }^{\circledR}$ for detection of A. baumannii in comparison to SBA supplemented with ertapenem. 


\section{Materials and methods}

We conducted this study from March to June 2012 at Sakarya University, School of Medicine Hospital, Sakarya, Turkey. In total, 73 Gram-negative bacteria were included in the study. The isolates consisted of $20 \mathrm{~A}$. baumannii (detection of OXA51, OXA23 and OXA58 genes was done by in-house polymerase chain reaction (PCR) 20 Pseudomonas aeruginosa (carbapenem-susceptible and -resistant), 15 Klebsiella pneumoniae (KPC carbapenemase-positive and negative), 10 Escherichia coli and five Stenotrophomonas maltophilia. The strains were single (one per patient) clinical isolates recovered from patients who were hospitalised in various clinics. The isolates were identified using conventional techniques (colony morphology, oxidase, and other biochemical tests) as well as the Vitek 2 system using N91 and N90 cassettes (bioMerieux, France).

Two different media were evaluated for their ability to distinguish A. baumannii from other bacteria. Twenty mixed cultures of four carbapenem-susceptible isolates (A. baumannii, P. aeruginosa, $K$. pneumonia, and E. coli) and Candida albicans were suspended in $0.9 \%$ sterile saline. A $1-\mu \mathrm{m}$ sterile loop dipper was used to inoculate CHROMagar Acinetobacter ${ }^{\circledR}$ and supplemented SBA plates at a density of $10^{6}$ colony forming units (CFU). Plates were scored by two independent observers blinded to the inoculum composition for the presence of $A$. baumannii. Colonies thought to be $A$. baumannii were confirmed using the Vitek 2 system. Colony counts were conducted to verify inoculum densities.

CHROMagar Acinetobacter ${ }^{\circledR}$ and SBA (Oxoid, England) were prepared from dehydrated powder according to the manufacturer's instructions. CHROMagar Acinetobacter ${ }^{\circledR}$ was prepared without and with supplementation with KPC (CHROMagar, Paris, France) at concentrations of 400 and $800 \mathrm{mg} / \mathrm{L}$. KPC was added according to the manufacturer's instructions in the CHROMagar Acinetobacter ${ }^{\circledR}$ at $50^{\circ} \mathrm{C}$. SBA was supplemented with $2 \mathrm{mg} / \mathrm{L}$ ertapenem (Merck \& Co., USA) at $50^{\circ} \mathrm{C}$. Inoculated media were incubated at $37^{\circ} \mathrm{C}$ in aerobic conditions and evaluated after 18-24h. These media were examined for presumptive colonies in accordance with the manufacturer's recommendations. SBA medium cultures were evaluated with conventional methods, such as colony morphology, oxides, and haemolysis. Each medium batch was quality controlled using standard strains (P. aeruginosa ATCC 27853, E. coli ATCC 602357 and $K$. pneumoniae ATCC 700603) obtained from Oxoid. Medical ethical approval of this study was deemed unnecessary.

\section{Results}

A. baumannii, P. aeruginosa, S. maltophilia and KPC-positive $K$. pneumoniae were grown on CHROMagar Acinetobacter ${ }^{\circledR}$ and CHROMagar supplemented with KPC for $24 \mathrm{~h}$. KPC-positive $K$. pneumoniae were distinguishable by their colony morphology and blue colour. A. baumannii, P. aeruginosa and S. maltophilia colonies all appeared weakly red after the first $24 \mathrm{~h}$. However, other Gramnegative bacteria were easily distinguishable by their dark blue colour or lack of colour, and small colony morphology after $48 \mathrm{~h}$. Susceptible clinical isolates and standard Enterobacteriaceae strains were inhibited by both CHROMagar Acinetobacter ${ }^{\mathbb{B}}$ and supplemented SBA. C. albicans was inhibited by CHROMagar Acinetobacter ${ }^{\mathbb{B}}$, but formed typical colonies on supplemented SBA. Growth results using CHROMagar Acinetobacter ${ }^{\circledR}$ supplemented with KPC are summarised in the Table 1 and Figure 1.

Table I Comparison of CHROMagar Acinetobacter with SBA medium for selection of A. baumannii and other multidrug-resistant isolates

\begin{tabular}{|c|c|c|c|c|c|c|c|c|c|c|}
\hline Isolate & $\mathbf{n}$ & $\begin{array}{l}\text { CHR } \\
24 \mathrm{~h}\end{array}$ & $\mathrm{CHR}^{+}$ & $\mathrm{CHR}^{++}$ & SBA $^{+}$ & $\begin{array}{l}\text { CHR } \\
48 \mathrm{~h} \\
\end{array}$ & $\mathrm{CHR}^{+}$ & $\mathrm{CHR}^{++}$ & SBA $^{+}$ & CHROMagar Colony Colour \\
\hline 'A. baumannii & 10 & 10 & 6 & $4 *$ & 10 & 10 & 7 & $6^{*}$ & 10 & Reddish \\
\hline${ }^{2} \mathrm{OXA} 23+\mathrm{A} \cdot \mathrm{bau}$ & 5 & 5 & 5 & 5 & 5 & 5 & 5 & 5 & 5 & Reddish \\
\hline${ }^{2} \mathrm{OXA} 58+\mathrm{A} \cdot \mathrm{bau}$ & 5 & 5 & 5 & 5 & 5 & 5 & 5 & 5 & 5 & Reddish \\
\hline 'P. aeruginosa & 10 & 10 & 5 & $3 *$ & 10 & 10 & 8 & $6 *$ & 10 & Reddish \\
\hline${ }^{2} P$. aeruginosa & 10 & 10 & 10 & 10 & 10 & 10 & 10 & 10 & 10 & Reddish \\
\hline S. maltophilia & 5 & 5 & $4^{*}$ & - & $5 *$ & 5 & $5^{*}$ & $2 *$ & $5^{*}$ & Reddish \\
\hline 'K. pneumoniae & 5 & $3^{*}$ & - & - & - & $5^{*}$ & - & - & - & Blue \\
\hline${ }^{2}$ K. pneumonia & 10 & 10 & 10 & 10 & 10 & 10 & 10 & 10 & 10 & Blue \\
\hline 'E. coli & 10 & $2^{*}$ & - & - & - & $3 *$ & - & - & - & Blue \\
\hline${ }^{\prime}$ C. albicans & 1 & - & - & - & I & - & - & - & I & - \\
\hline P. aer ATCC27853 & 1 & I & - & - & I & 1 & - & - & I & Reddish \\
\hline K. pne ATCC700603 & I & - & - & - & - & - & - & - & - & Blue \\
\hline E. coli ATCC 602357 & I & - & - & - & - & - & - & - & - & Blue \\
\hline
\end{tabular}

*Small colonies and/or low colony count,

'Susceptible clinical isolate,

${ }^{2}$ Carbapenem-resistant clinical isolate; MIC, $>32 \mu \mathrm{L} / \mathrm{mL}$.

CHR, CHROM Agar Acinetobacter; $\mathrm{CHR}^{+}, \mathrm{CHROM}$ Agar Acinetobacter Supplemented With 400 mg/L KPC; CHR ${ }^{++}$, CHROM Agar Acinetobacter Supplemented With 800 mg/L KPC; SBA ${ }^{+}$, Sheep Blood Agar Supplemented With 2 mg/L ertapenem 


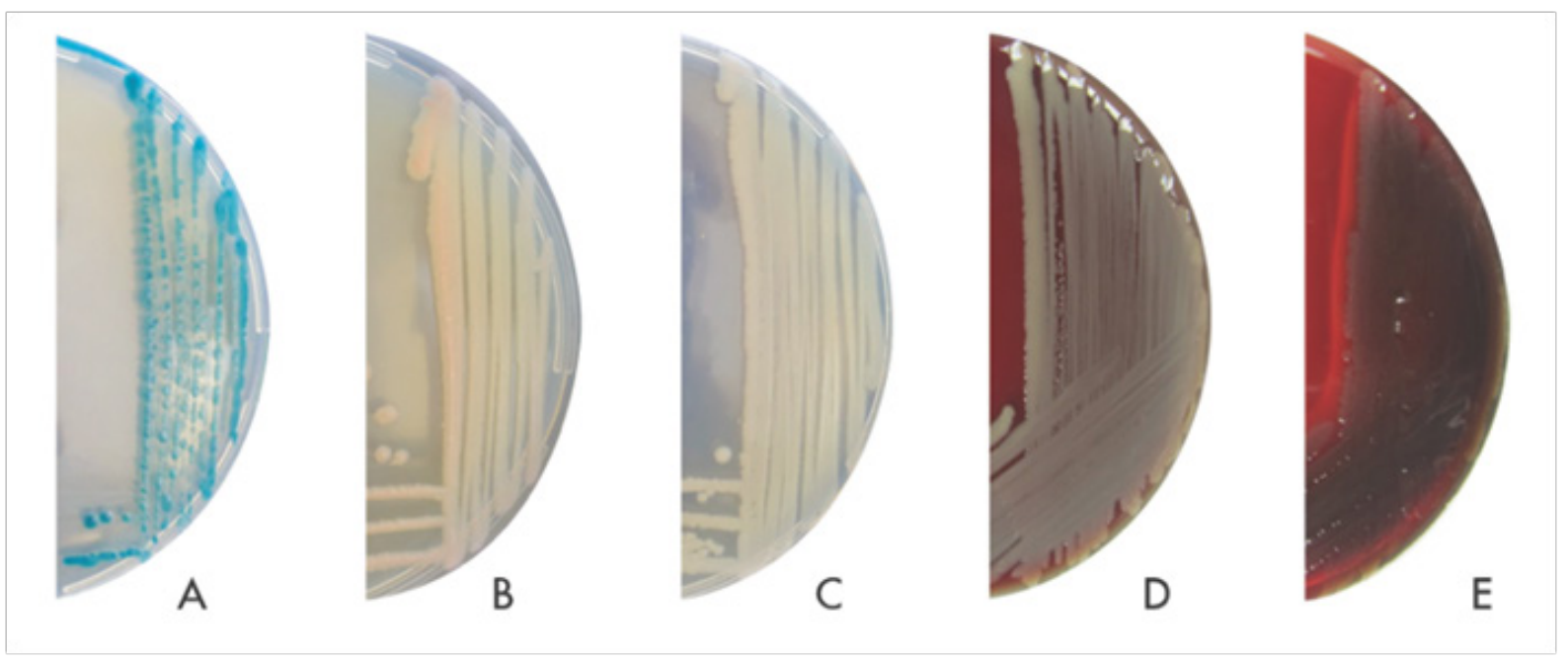

Figure I Cultured bacteria view in SBA and CHROMagar Acinetobacter.

A, Klebsiella pneumonia in CHROMagar Acinetobacter; B, Acinetobacter baumannii in CHROMagar Acinetobacter; C, Pseudomonas aeruginosa in CHROMagar Acinetobacter; D, Acinetobacter baumannii in SBA; E, Pseudomonas aeruginosa in SBA

A. baumannii, P. aeruginosa, S. maltophilia, KPC-positive $K$. pneumoniae and C. albicans were grown on supplemented SBA for $24 \mathrm{~h}$. Other Gram-negative bacteria did not grow on supplemented SBA after 24 or 48 h. A. baumannii grown on supplemented SBA were recognisable by their smooth colony morphology, were nonhaemolytic and were colourless after $24 \mathrm{~h}$. P. aeruginosa isolates grown on supplemented SBA were distinct in their colony morphology, colour and demonstration of haemolysis after $24 \mathrm{~h}$. S. maltophilia were not recognisable by their appearance, as they formed small colonies in the first $24 \mathrm{~h}$. Moreover, KPC-positive $K$. pneumoniae isolates were non-haemolytic, colourless and had mucoid colonies after growth on supplemented SBA for $24 \mathrm{~h}$.

A. baumannii, P. aeruginosa, S. maltophilia and KPC-positive $K$. pneumoniae isolates, which were inoculated as mixed samples, were grown on CHROMagar Acinetobacter ${ }^{\circledR}$ for 24 h. P. aeruginosa and $S$. maltophilia colonies were the same colour as those of $A$. baumannii, and the $S$. maltophilia colonies were small. C. albicans isolates, inoculated with mixed samples, did not grow on CHROMagar Acinetobacter ${ }^{\circledR}$ after 24 or $48 \mathrm{~h}$, but did grow on supplemented SBA after $24 \mathrm{~h}$ and exhibited distinct colony morphology.

\section{Discussion}

A. baumannii the most clinically relevant of the bacteria tested, have a particular propensity for nosocomial transmission, due in part to their sustained survival on environmental surfaces as well as their multidrug resistance. The prevalence of infection with A. baumannii has increased significantly during the last decade. Moreover, $A$. baumannii have developed one of the most impressive patterns of antibiotic resistance ever observed. Therefore, rapid detection of $A$. baumannii in clinical samples could improve the targeted implementation of infection control measures and potentially aid the selection of empirical therapies for infected patients.,

To culture A. baumannii isolated from clinical samples and to screen for carriers among intensive care unit patients, use of a selective enrichment medium is recommended. In recent years, a few previous reports about CHROMagar Acinetobacter ${ }^{\circledR}$ have been published with regard to multidrug resistant A. baumannii (MDRAB). Gordon et al., ${ }^{6}$ reported that the availability of selective culture media, such as CHROMagar Acinetobacter ${ }^{\mathbb{R}}$, enabled those who may benefit from such technology to be identified rapidly. Their findings supported the use of CHROMagar Acinetobacter ${ }^{\mathbb{B}}$ as a (limited) means of identifying the presence of enteric MDRAB. Using a well-characterised panel of carbapenem-susceptible and -resistant $A$. baumannii isolates grown in CHROMagar Acinetobacter ${ }^{\mathbb{R}}$, selectivity for carbapenem-resistant A. baumannii was not demonstrated. ${ }^{7}$ In addition, some isolates of Staphylococcus aureus, P. aeruginosa and Enterococcus cloacae formed colonies of the same colour as A. baumannii, suggesting the possibility of misdiagnosis. Panagea et al., ${ }^{8}$ made a similar observation and stated that CHROMagar KPC medium was a useful screening medium both for KPC and VIM carbapenemase-producing Enterobacteriaceae in stool samples.

Our data suggest that neither supplemented nor non-supplemented CHROMagar Acinetobacter ${ }^{\mathbb{B}}$ media differed from supplemented SBA in terms of selection of $A$. baumannii. Definitive identification of bacteria grown in either selective medium may require additional testing. A. baumannii and $P$. aeruginosa could be differentiated by performing an oxidase test. Supplemented SBA had greater discriminatory power than CHROMagar Acinetobacter $^{\circledR}$ for $P$. aeruginosa; however, CHROMagar Acinetobacter ${ }^{\circledR}$ proved to be a reliable rapid-isolation medium for non-fermentative bacteria. Growth of most other Gram-negative bacteria and yeasts was inhibited. CHROMagar Acinetobacter ${ }^{\circledR}$ cannot be recommended as the sole medium for isolation and screening of $A$. baumannii. Further development of selective media is needed to enable identification of A. baumannii or MDRAB. Selective culture media are increasingly necessary due to the exponential increase in the incidence of $A$. baumannii infection. Use of media selective for $A$. baumannii will enable rapid identification.

\section{Conclusion}

In conclusion, detection of resistance is crucial for infection control policies and the availability of cost-effective reagents will result in more rapid identification of infected patients compared to 
other conventional techniques, permitting immediate implementation of infection control measures to prevent further dissemination. Such measures direct therapy away from inappropriate antibiotics. Welldesigned solid, selective culture media thus remain necessary. We advise the use of supplemented SBA and CHROMagar Acinetobacter ${ }^{\circledR}$ medium for A. baumannii for rapid detection of nosocomial infections in the absence of confirmatory procedures. Investigation into the metabolism of $A$. baumannii will facilitate the development of selective agars.

\section{Acknowledgments}

This study is an experimental study. In this study in-vitro samples were investigated retrospectively. Samples are not taken from patient.

\section{Conflicts of interest}

Authors declare that there is no conflict of interest.

\section{References}

1. Dijkshoorn L, Nemec A. The diversity of the genus Acinetobacter. In: Gerischer U (Ed.), Acinetobacter Molecular Biology. Caister Academic Press: UK; 2008. 1-34 p.

2. Villegas MV, Hartstein AI. Acinetobacter outbreaks, 1977-2000. Infect Control Hosp Epidemiol. 2003;24(4):284-295.
3. Towner KJ. Molecular basis of antibiotic resistance in Acinetobacter spp. In: Gerischer U (Ed.), Acinetobacter Molecular Biology. Caister Academic Press: UK; 2008. 321-343 p.

4. Gordon NC, Wareham DW. Multidrug-resistant Acinetobacter baumannii: mechanisms of virulence and resistance. Int $J$ Antimicrob Agents. 2010;35(3):219-226.

5. Valentine SC, Contreras D, Tan S, et al. Phenotypic and molecular characterization of Acinetobacter baumannii clinical isolates from nosocomial outbreaks in Los Angeles County, California. J Clin Microbiol. 2008;46(8):2499-2507.

6. Gordon NC, Wareham DW. Evaluation of CHROMagar Acinetobacter for detection of enteric carriage of multidrug-resistant Acinetobacter baumannii in samples from critically ill patients. J Clin Microbiol. 2009;47(7):2249-2251.

7. Akers KS, Barsoumian A, Beckius ML, et al. CHROMagar Acinetobacter is not selective for carbapenem resistant Acinetobacter baumanniicalcoaceticus complex. Diagn Microbiol Infect Dis. 2010;67(2):209-211.

8. Panagea T, Galani I, Souli M, et al. Evaluation of CHROMagar ${ }^{\mathrm{TM}}$ KPC for the detection of carbapenemase-producing Enterobacteriaceae in rectal surveillance cultures. Int J Antimicrob Agents. 2011;37(2):124-128. 